\title{
Variable wave vector second harmonic generation in phenanthrene ${ }^{a)}$
}

\author{
Carey K. Johnson and Gerald J. Small \\ Ames Laboratory and Department of Chemistry, Iowa State University, Ames, Iowa 50011 \\ (Received 14 September 1981; accepted 30 November 1981)
}

The flexibility inherent in nonlinear spectroscopic techniques has provided the means to investigate the wave-vector dependence of the energies of mixed exciton-photon states (polaritons) in organic crystals. In the polariton theory, two-photon absorption (TPA) and second harmonic generation (SHG) to or via states strongly coupled to the radiation field are understood to proceed by the fusion ${ }^{1,2}$ of two polaritons to form a frequency-doubled polariton. The conservation of crystal momentum during polariton fusion requires phase matching. ${ }^{3}$ Hochstrasser and Meredith used angle tuning of resonant phase-matched SHG to demonstrate polariton dispersion in anthracene and naphthalene. ${ }^{4}$ Very recently, the competition between TPA and SHG, implicit in the polariton fusion model, was studied in sublimed naphthalene crystals through variation of the temperature-dependent polariton-phonon scattering. ${ }^{5}$

The phenanthrene crystal's $350 \mathrm{~nm}$ origin transition is comprised of two Davydov components, the $a$-polarized exciton at $28597 \mathrm{~cm}^{-1}$ and the $b$ exciton at $28656 \mathrm{~cm}^{-1}, 6$ referred to as the lower and upper Davydov components. We have reported the dependence of the phase matching on the upper branch of the $b$ polariton on photon propagation direction and polarization for two-photon excitation (TPE) and SHG in the phenanthrene origin region. ${ }^{7,8} \mathrm{We}$ report here additional evidence to support this interpretation. In this experiment, the dye-laser beam was split into two beams which intersect in the crystal at an

c'(bb) crossed-beam SHG

$13^{\circ}$ $21^{\circ}$
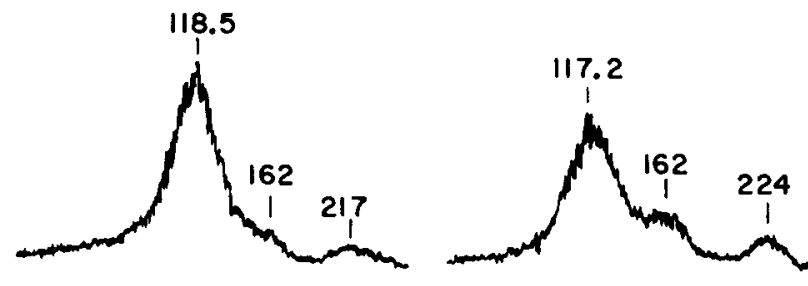

$28^{\circ}$ $37^{\circ}$

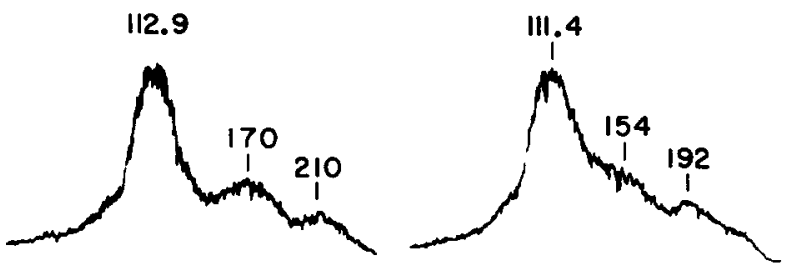

FIG. 1. Dependence of the phase-matched SHG for the $b$ polariton on the angle between incident beams observed by crossed-beam generation of the $b$-polarized second harmonic by light incident on the $a b$ face of sublimed phenanthrene at $1.8 \mathrm{~K}$. angle $\theta$. If one photon from each beam participates in a two-photon process, the wave vector formed $k_{1}+k_{2}$ depends on $\theta$. By varying $\theta$ it is possible to vary the phase-matching point on the polariton dispersion curve. This experiment has been done in inorganic crystals by TPA $^{1,9}$ and SHG, ${ }^{10}$ but apparently has not been reported previously for molecular crystals. The experimental system, described elsewhere, "11 allows the simultaneous detection of SHG and TPE. The photomultiplier for SHG detection was positioned so that only the beam formed by the vector sum $k_{1}+k_{2}$ was detected, while beams collinear with $k_{1}$ or $k_{2}$ were blocked. The TPE signal was the sum of TPE from one or both beams. The beams were incident on the $a b$ face of a sublimed phenanthrene crystal at 1.6 to $1.8 \mathrm{~K}$.

With both beams polarized $\| b$, the polariton branches associated with the upper Davydov component are reached by polariton fusion. ${ }^{7}$ The resulting SHG signal was $b$ polarized, in accord with a factor-group analysis. ${ }^{7,8}$ As shown in Fig. 1, the SHG maximum decreases from 118 (relative to the lower component) to $111 \mathrm{~cm}^{-1}$ as $\theta$ is increased from $13^{\circ}$ to $37^{\circ}$, tuning the phase-matching point on the upper branch of the polariton dispersion curve. This accords with the interpretation of shifts of the upper Davydov component in TPE and SHG. ${ }^{7}$ The decrease in the position of the upper component with increasing $\theta$ is approximately that expected based on the polariton dispersion curve for the approximately $2 \%$ decrease in $\left|k_{1}+k_{2}\right|$ inside the crystal as $\theta$ is varied from $13^{\circ}$ to $37^{\circ}$ outside the crystal. ${ }^{11}$ The structure apparent above the principal SHG maximum in Fig. 1 has been attributed to exciton-phonon states. ${ }^{8}$

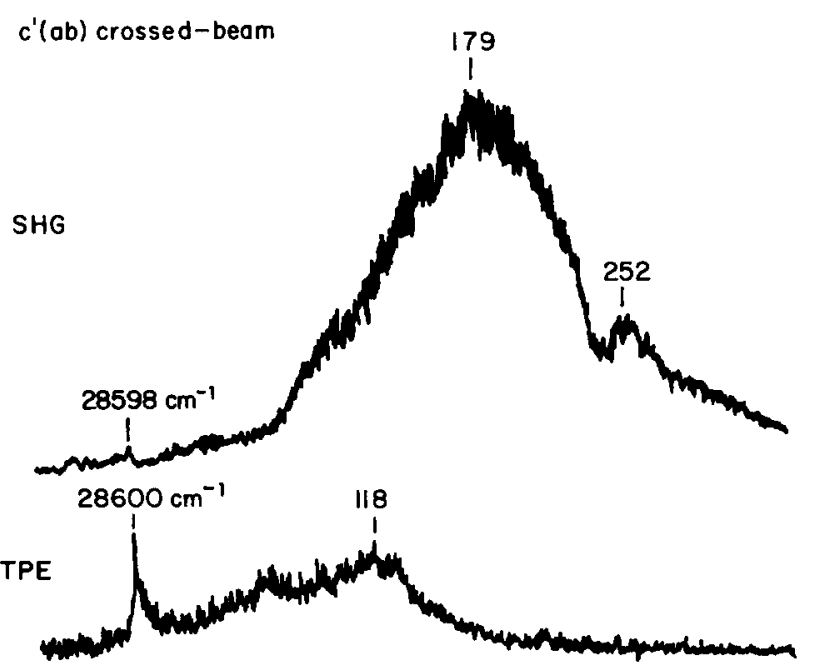

FIG. 2. Crossed-beam $a$-polarized SHG and TPE for $a$ - and $b$-polarized light incident at an angle of $13^{\circ}$ relative to one anothe $r$ on the $a b$ face of sublimed phenanthrene at $1.6 \mathrm{~K}$. 
When one of the incoming beams was polarized $\perp b, a$ polarized SHG was observed, resulting from the polariton associated with the $a$ exciton. At different $\theta$, the peak in SHG intensity was observed to vary from about 110 to $205 \mathrm{~cm}^{-1}$ above the $a$ exciton. The experiment for $a$-polarized SHG was complicated by the fact that one incoming beam and the outgoing beam were extraordinary rays. Thus, $\left|k_{1}+k_{2}\right|$ depended not only on $\theta$, but also on the refractive indicies of the extraordinary rays. This was verified by rotating the crystal about the $b$ axis at a given $\theta$. The phase-matching point was found to vary by as much as $50 \mathrm{~cm}^{-1}$.

A representative crossed-beam $a$-polarized SHG spectrum is shown in Fig. 2 along with the associated TPE spectrum. The SHG is observed some $180 \mathrm{~cm}^{-1}$ above the $a$ exciton, demonstrating phase matching to the elbow region of the upper branch of the $a$ polariton. (The elbow region of the $a$-polariton's upper branch is calculated to lie 100 to $200 \mathrm{~cm}^{-1}$ above the transverse $a$ exciton. ${ }^{2.11}$ ) The feature at $\sim 118 \mathrm{~cm}^{-1}$ in TPE is the $b$ polariton, excited by two photons of the same polarization. The shoulder at $252 \mathrm{~cm}^{-1}$ in SHG is due to a fundamental vibration. ${ }^{8}$ We have suggested ${ }^{7}$ that the TPE lower component at $28600 \mathrm{~cm}^{-1}$ in TPE might be due to the longitudinal exciton of the lower Davydov component. As such, it cannot and does not appear in SHG. However, in several spectra, a weak feature was observed in SHG, appearing consistently at 2 to $4 \mathrm{~cm}^{-1}$ below the TPE lower component. This is likely resonant SHG at the frequency of the transverse $a$ exciton (Philpott and Syassen ${ }^{12}$ estimate the stop gap as $\sim 4 \mathrm{~cm}^{-1}$ for the $a$ polariton). The absence of the feature at $28600 \mathrm{~cm}^{-1}$ in SHG supports the suggestion that the lower component appearing in TPE is a longitudinal exciton.

a) This work was supported by the Director for Energy Research, Office of Basic Energy Science, U.S. Department of Energy operated by Iowa State University under contract No. W-7405-Eng-82.

${ }^{1} \mathrm{D}$. Fröhlich, E. Mohler, and $\mathrm{Ch}$. Uihlein, Phys. Status Solidi B 55, 175 (1973).

${ }^{2}$ C. K. Johnson and G. J. Small, in Excited States, edited by B. C. Lim (Academic, New York, in press), Vol. 6 .

${ }^{3}$ L. N. Ovander, Usp. Fiz. Nauk 86, 3 (1965); [Sov. Phys. Usp. (Engl. Transl.) 8, 337 (1965) ].

${ }^{4}$ R. M. Hochstrasser and G. R. Meredith, J. Chem. Phys. 67, 1273 (1977).

${ }^{5}$ S. H. Stevenson, C. K. Johnson, and G. J. Small, J. Phys. Chem. 85, 2709 (1981).

${ }^{6}$ D. P. Craig and R. D. Gordon, Proc. R. Soc. London Ser. A 288, 69 (1965).

${ }^{7}$ C. K. Johnson and G. J. Small, Chem. Phys. 64, 83 (1982).

${ }^{8}$ V. SethuRaman, C. K. Johnson, C. SethuRaman, and G. J. Small, Mol. Cryst. Liq. Cryst. 57, 89 (1980).

D. Fröhlich, E. Mohler, and P. Weisner, Phys. Rev, Lett. 26, 554 (1971).

10D. C. Haueisen and H. Mahr, Phys. Lett. A 36, 433 (1971); Phys, Rev, B 8, 2969 (1973).

${ }^{11}$ Carey K. Johnson, Ph.D. thesis, Iowa State University, 1981.

${ }^{12}$ K. Syassen and M. R. Philpott, J. Chem. Phys. 69, 1251 (1978).

\title{
Morse-oscillator matrix elements appropriate for vibration-rotation intensities of diatomic molecules
}

\author{
J. N. Huffaker and Loc Binh Tran \\ Department of Physics and Astronomy, Oklahoma University, Norman, Oklahoma 73019 \\ (Received 26 March 1981; accepted 22 April 1981)
}

In analyzing intensities of vibration-rotation transitions in diatomic molecules, one usually assumes a parametric form for the electric dipole function $M(r)$ as a function of internuclear separation and then tries to determine the parameters by a fit to measured intensities. Thus, Heaps and Herzberg ${ }^{1}$ fit intensities in $\mathrm{OH}$ using for $M(r)$ linear and quadratic functions of $q=r-r_{e}$. For this purpose, they calculated matrix elements of $q$ and $q^{2}$ between levels of a Morse oscillator. Herman and Rubin ${ }^{2}$ presented formulas from which matrix elements of higher powers of $q$ can be extracted. This procedure is also extended to the analysis of band intensities in electronic transitions. ${ }^{2,3}$

The perturbed-Morse-oscillator (PMO) potential, ${ }^{4}$ which is a series in powers of the quantity

$$
y=1-\exp (-a q),
$$

has proven to be an extremely accurate model for the effective vibrational potential of diatomic molecules. With its excellent convergence properties and good asymptotic behavior, it clearly outperforms the Dunham potential, ${ }^{5,6}$ an expansion in powers of $q$. Perhaps sim- ilar advantages might come from expanding $M(r)$ in powers of $y$ instead of powers of $q .^{7}$

We have calculated formulas for matrix elements of $y, y^{2}, y^{3}$, and $y^{4}$ for bound levels of a Morse oscillator, using standard techniques of the factorization method. ${ }^{8}$ These are rather simple algebraic formulas, which will be easy to apply to either hand or machine computation. Diagonal matrix elements are given by

$$
\begin{aligned}
& \langle v|y| v\rangle=\left\langle v\left|y^{2}\right| v\right\rangle=u / \sigma, \\
& \left\langle v\left|y^{3}\right| v\right\rangle=\left(6 u^{2}+\frac{3}{2}\right) / s^{2}-\left(4 u^{3}+3 u\right) / s^{3}, \\
& \left\langle v\left|y^{4}\right| v\right\rangle=\left(6 u^{2}+\frac{3}{2}\right) / s^{2}-\left(4 u^{3}+3 u\right) / s^{3},
\end{aligned}
$$

where $u=v+\frac{1}{2}, S=2 \sigma$, and $\sigma=\left(2 \mu V_{e}\right)^{1 / 2} /(a \hbar)$ (see paper I). Results in Eq. (2a) were published previously. ${ }^{9}$

Formulas for off-diagonal matrix elements contain phase factors which must be fixed by convention. Two common conventions for Morse eigenfunctions are as follows: (1) Every eigenfunction is positive at its inner "classical turning point" as produced by a raising operator of class II, type B factorization. ${ }^{8}$ (2) Every eigenfunction is positive at its outer "classical turning 